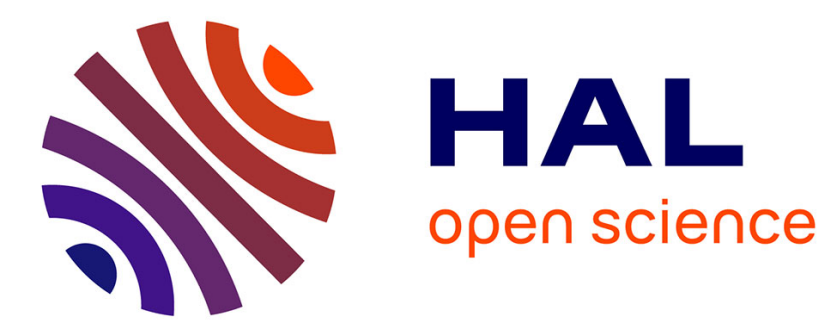

\title{
Thermochimie solaire à hautes températures, résultats expérimentaux. Quelques perspectives d'application
}

\author{
G. Flamant
}

\section{To cite this version:}

G. Flamant. Thermochimie solaire à hautes températures, résultats expérimentaux. Quelques perspectives d'application. Revue de Physique Appliquée, 1980, 15 (3), pp.503-511. 10.1051/rphysap:01980001503050300 . jpa-00244755

HAL Id: jpa-00244755

https://hal.science/jpa-00244755

Submitted on 1 Jan 1980

HAL is a multi-disciplinary open access archive for the deposit and dissemination of scientific research documents, whether they are published or not. The documents may come from teaching and research institutions in France or abroad, or from public or private research centers.
L'archive ouverte pluridisciplinaire HAL, est destinée au dépôt et à la diffusion de documents scientifiques de niveau recherche, publiés ou non, émanant des établissements d'enseignement et de recherche français ou étrangers, des laboratoires publics ou privés. 


\title{
Thermochimie solaire à hautes températures, résultats expérimentaux. Quelques perspectives d'application
}

\author{
G. Flamant \\ Laboratoire d’énergétique solaire, BP 5, Odeillo, 66120 Font Romeu, France
}

(Reçu le 10 septembre 1979, révisé le 19 novembre 1979, accepté le 19 novembre 1979)

\begin{abstract}
Résumé. - A partir d'expériences réalisées à l'aide de petits fours solaires de $2 \mathrm{~kW}$ de puissance concernant : i) le chauffage entre 600 et $1300^{\circ} \mathrm{C}$ de particules réfractaires; ii) la décarbonatation de $\mathrm{CaCO}_{3}$ à $850^{\circ} \mathrm{C}$, l'auteur suggère quelques applications à l'échelle industrielle des réacteurs étudiés dans les domaines du stockage thermique ou chimique de l'énergie et de la chimie préparative des liants hydrauliques. Le réacteur à lit fluidisé et le four rotatif présentent en effet des caractéristiques intéressantes pour la réalisation de réactions gaz-solide vers $900{ }^{\circ} \mathrm{C}$ par l'énergie solaire concentrée.
\end{abstract}

\begin{abstract}
Some large scale applications of solar energy at about $900^{\circ} \mathrm{C}$ are proposed, in the field of thermal or chemical storage of energy and the industrial production of lime or portland cement, using fluidized bed reactor or rotary kiln. This type of chemical reactor has been studied with small solar furnaces (power : $\sim 2 \mathrm{~kW}$ ). They allow continuous processing at high temperature eg : i) heating of refractory materials at $600{ }^{\circ} \mathrm{C}-1300{ }^{\circ} \mathrm{C}$; ii) decarbonation of calcite at $850^{\circ} \mathrm{C}$.
\end{abstract}

Introduction. - Environ $10 \%$ de l'énergie consommée en France sert à effectuer des traitements thermiques à plus de $600^{\circ} \mathrm{C}$ dans l'industrie. Parallèlement, la chaleur solaire à haute température semble être un mode de valorisation de nos ressources naturelles thermodynamiquement et bientôt économiquement viable.

Parmi ces procédés industriels, les réactions gazsolide (décompositions, réactions catalytiques,...) constituent un domaine d'application de l'énergie solaire concentré particulièrement intéressant, mais nécessite le développement d'un génie chimique adapté [21].

$\mathrm{Au}$ cours d'une étude réalisée au laboratoire des ultra-réfractaires d'Odeillo, en collaboration avec l'équipe de recherche sur l'énergie de l'Université Paul-Sabatier de Toulouse $[9,10]$, nous nous sommes attachés à définir les conditions expérimentales optimales en vue : $i$ ) du chauffage de solides réfractaires divisés, ii) de la décarbonatation de la calcite à l'aide de petits fours solaires de $2 \mathrm{~kW}$ environ. Nous rappelons dans une première partie les résultats essentiels de cette étude avant de proposer quelques développements possibles des réacteurs mis en œuvre.

1. Réacteurs solaires à lit fluidisé et à lit brassé (four rotatif). - 1.1 DESCRIPTION DES RÉACTEURS. - Les deux réacteurs de laboratoire étudiés sont schématisés sur les figures 1 et 2 .
Associé à un four solaire de type parabolique à axe vertical (puissance utile $P_{\mathrm{u}}=1,4 \mathrm{~kW}$ ), le lit fluidisé (Fig. 1) est constitué, pour l'essentiel. d'un tube en silice transparente de diamètre $34 \mathrm{~mm}$ [5] disposé entre deux pièces métalliques où sont fixées les diverses sondes $-6,7,8,10-$. Les rayons solaires concentrés interceptent la surface supérieure du lit. Le rayonnement réfléchi et émis est partiellement renvoyé vers les particules à l'aide de réflecteurs entourant la colonne.

Le four rotatif (Fig. 2), incliné de $5^{\circ}$ par rapport à l'horizontale, est composé d'une capacité métallique réfrigérée par circulation d'eau - 7 - dans laquelle est placé un tube réfractaire $(\varnothing=20$ ou $24 \mathrm{~mm}, L=90 \mathrm{~mm}$ ) -1 - en alumine ou en chromite de lanthane. L'ensemble est mis en rotation à vitesse variable et fonctionne en régime continu. Les temps de séjour des particules varient de $20 \mathrm{~s}(\omega=25 \mathrm{tr} / \mathrm{min})$, à $120 \mathrm{~s}(\omega=4 \mathrm{tr} / \mathrm{min})$. Les températures sont mesurées soit par thermocouples, soit par pyrométrie optique dans le proche infrarouge $(2,9 \mu \mathrm{m})$.

1.2 Niveau DE TEMPÉRATURE ATtEINT AVEC DES CHARGES INERTES ET DISTRIBUTION VOLUMIQUE DES TEMPÉRATURES. - Les couches fluidisées absorbant directement le rayonnement, le niveau de température atteint à l'équilibre par le système dépend en premier lieu de ses propriétés d'absorption et du facteur d'absorption solaire des particules en particulier. 


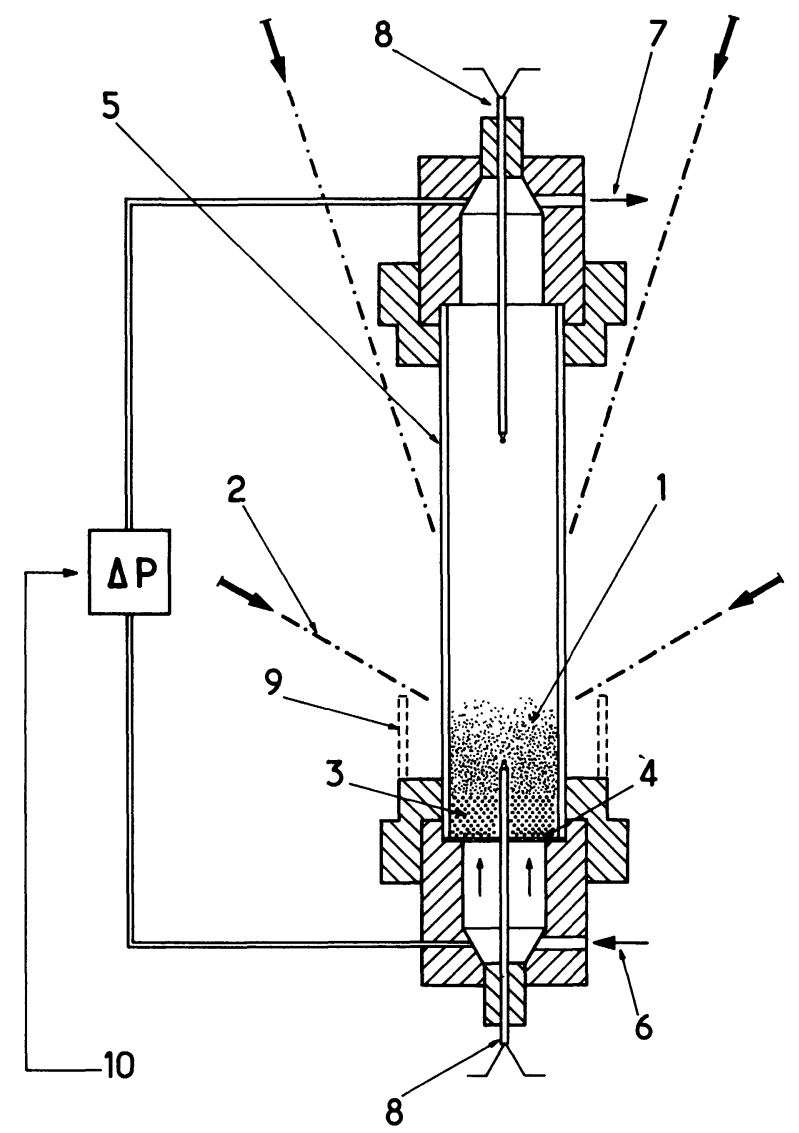

Fig. 1. - Représentation schématique du réacteur à lit fluidisé (échelle environ 0,25). 1 : Couche fluidisée. 2 : Rayonnement solaire concentré. 3 : Distributeur à billes. 4 : Grille support. 5 : Tube en silice transparente. 6 : Entrée du gaz vecteur. 7 : Sortie gaz. 8 : Thermocouples. 9 : Réflecteur. 10 : Mesure de la perte de charge.

[Scheme of the solar fluidized bed reactor. 1 : Fluid bed. 2 : Concentrated solar rays. 3 : Gas distributor consisting of glass, iron or zirconia beads. 4 : Grid. 5 : Transparent silica tube. 6: Gas inlet. 7 : Gas outlet. 8 : Thermocouples. 9 : Reflectors. 10 : Pressure loss measurement.]

Nous avons réuni dans le tableau I les températures moyennes des lits fluidisés obtenues à partir de sable naturel, chamotte $\left(55 \% \mathrm{SiO}_{2}, 40 \% \mathrm{Al}_{2} \mathrm{O}_{3}, \mathrm{Fe}_{2} \mathrm{O}_{3}\right.$, $\left.\mathrm{TiO}_{2}\right)$, ilménite $\left(20,6 \% \mathrm{FeO}, 20,4 \% \mathrm{Fe}_{2} \mathrm{O}_{3}, 54 \%\right.$ $\mathrm{TiO}_{2}, \mathrm{MnO}, \mathrm{Al}_{2} \mathrm{O}_{3}$ ) pour des diamètres de grains de 200-315 $\mu \mathrm{m}$ et des charges de $50 \mathrm{~g}$ (cf. Fig. 3).

On remarque l'accroissement du niveau de température résultant d'une augmentation du facteur d'absorption solaire des particules. Ce dernier a été mesuré au CERT de Toulouse, par réflectométrie à $20^{\circ} \mathrm{C}$, sur des échantillons de poudre tassée de même granulométrie que la charge en utilisant une lampe au xénon comme source.

En ce qui concerne la distribution volumique des températures, la figure 4 donne un exemple du profil axial de température dans la colonne de fluidisation. Par ailleurs, les mesures par thermocouple réalisées en différents points d'une section droite des couches fluidisées révèlent des écarts très faibles : inférieurs à $40^{\circ} \mathrm{C}$ entre 700 et $900^{\circ} \mathrm{C}$.
Tableau I. - Comparaison des résultats expérimentaux obtenus pour différentes couches fluidisées inertes. Vitesse superficielle du gaz $\left(\mathrm{N}_{2}\right): 0,05 \mathrm{~m} / \mathrm{s}$, puissance solaire utile : $1,4 \mathrm{~kW}$.

\begin{tabular}{|c|c|c|c|}
\hline Matériau & $\begin{array}{l}\text { Sable } \\
\text { naturel } \\
-\end{array}$ & $\begin{array}{c}\text { Chamotte } \\
-\end{array}$ & Ilménite \\
\hline $\begin{array}{l}\text { Facteur d'absorption so- } \\
\text { laire des particules }\left(\alpha_{s}\right)\end{array}$ & $0,4 \pm 0,06$ & $0,75 \pm 0,05$ & $0,95 \pm 0,05$ \\
\hline $\begin{array}{l}\text { Température d'équilibre } \\
\text { des couches fluidisées } \\
\left(T_{1}\right)\end{array}$ & & & \\
\hline $\begin{array}{l}\left(T_{1}\right) \\
\text { Capacité massique de } \\
\text { stockage thermique } \\
(\mathrm{kWh} / \mathrm{kg})\end{array}$ & $1000^{\circ} \mathrm{C}$ & $1100^{\circ} \mathrm{C}$ & $1250^{\circ} \mathrm{C}$ \\
\hline $\begin{array}{l}\text { Efficacite thermique du } \\
\text { transfert d'énergie au } \\
\text { solide }(\%)\end{array}$ & 16 & 25 & 35 \\
\hline $\begin{array}{l}\text { Fraction d'énergie trans- } \\
\text { férée au gaz }(\%)\end{array}$ & 3 & 4 & 5 \\
\hline
\end{tabular}

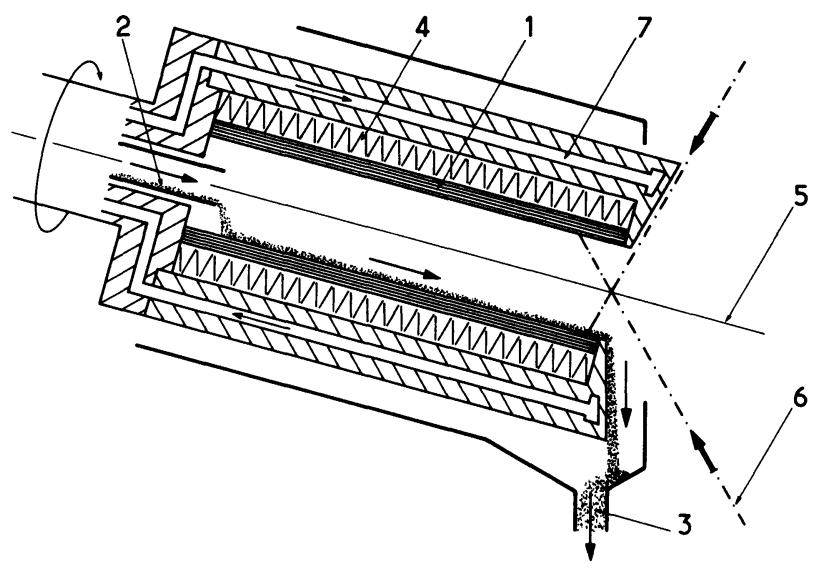

Fig. 2. - Représentation schématique du four rotatif : $1:$ Garnissage réfractaire. 2: Transporteur vibrant, alimentation en poudre. 3 : Sortie du solide. 4 : Isolant. 5 : Axe du four (inclinable). 6: Rayonnement solaire concentré. 7 : Paroi réfrigérée.

[Scheme of the solar rotary kiln : $1:$ Refractory tube $\left(\mathrm{Al}_{2} \mathrm{O}_{3}\right.$ or $\mathrm{LaCrO}_{3}$ ). 2 : Powder inlet. 3 : Powder outlet. 4. Insulator. $5:$ Axis of the kiln (may be tilted). 6 : Concentrated solar rays. $7:$ Water cooled metallic shell.]

Au contraire, les garnissages réfractaires du four rotatif présentent un gradient thermique important (environ $100^{\circ} \mathrm{C} / \mathrm{cm}$ ) sur toute leur longueur. La figure 5 donne un profil axial expérimental de température pour les deux tubes. La température de la charge à la sortie du four varie de 300 à $1500^{\circ} \mathrm{C}$ selon la nature du matériau et le débit massique. L'efficacité thermique $\left({ }^{1}\right)$ augmente avec le taux de

(1) L'efficacité thermique (chauffage de charges inertes) est définie par

$$
\eta=\frac{m C_{\mathrm{p}} \Delta T}{\Delta t P_{\mathrm{u}}} \text { ou } \frac{\dot{m} C_{\mathrm{p}} \Delta T}{P_{\mathrm{u}}}
$$

rapport de la chaleur massique de la charge - à la température d'équilibre $T$ - à l'énergie utile fournie par le four solaire pendant le temps $\Delta t$. 


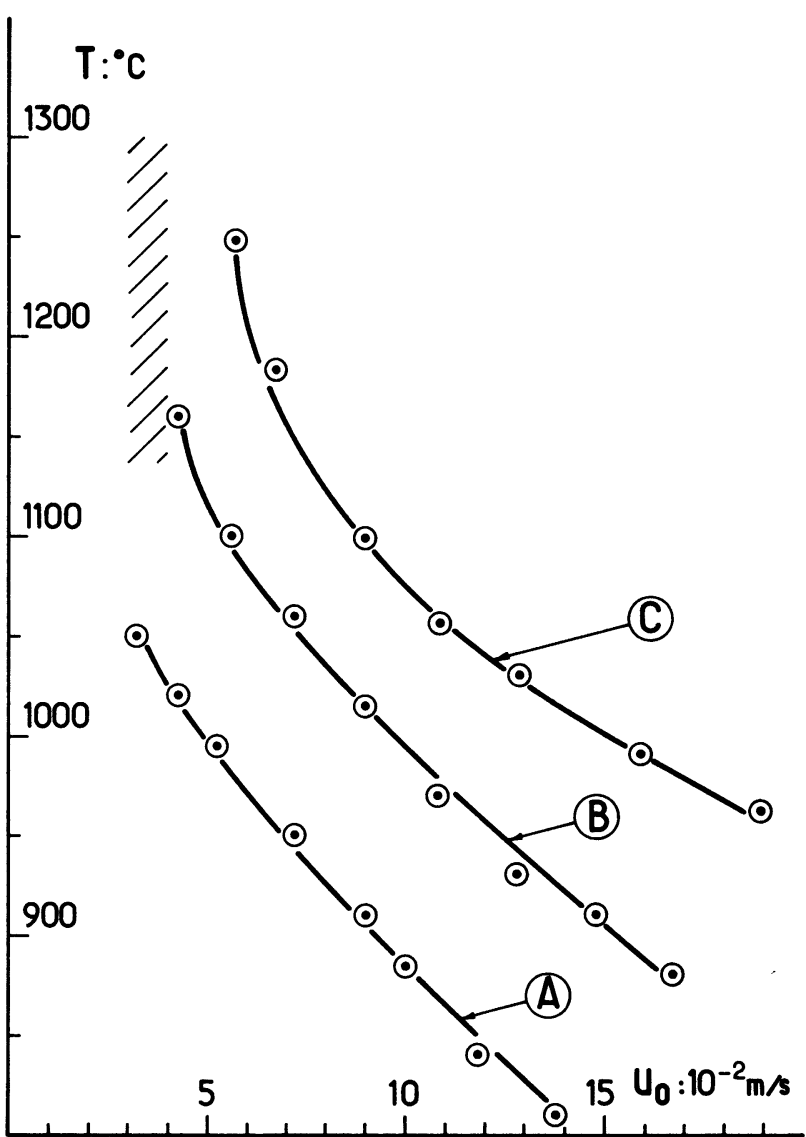

Fig. 3. - Température d'équilibre de couches fluidisées chauffées par rayonnement solaire en fonction de la vitesse superficielle du gaz. Puissance utile $P_{\mathrm{u}}=1,4 \mathrm{~kW}$, diamètre des grains 0,200 $0,315 \mathrm{~mm}$.

Courbe A : sable naturel, courbe B : chamotte, courbe C : ilménite.

[Measured temperature of fluidized materials (heated by means of concentrated solar energy) versus superficial gas velocity.

Particules diameter : $0.200-0.315 \mathrm{~mm}$, power : $1.4 \mathrm{~kW}$. Curves A : silice sand, B : chamotte, $\mathrm{C}$ : ilmenite.]

remplissage du four $\tau^{*}$ et atteint environ $30 \%$ (cf. Fig. 6).

1.3 Etude de la déCarbonatation de $\mathrm{CaCC}_{3}$. - L'étude de la décarbonatation de la calcite selon la réaction : $\mathrm{CaCO}_{3} \rightleftarrows \mathrm{CaO}+\mathrm{CO}_{2}$ est intéressante, tant au plan de la chimie préparative [9] qu'au plan du stockage chimique de l'énergie $[2,6,7]$.

En couche fluidisée et en régime discontinu, la vitesse de décarbonatation varie de 4 à $5,5 \mathrm{~g} / \mathrm{min}$ à $850^{\circ} \mathrm{C}$ selon la vitesse superficielle du gaz, le diamètre des grains, le distributeur de gaz vecteur qui sont utilisés. La décomposition totale est obtenue après 6 à $8 \mathrm{~min}$ et est accélérée par l'addition de poudre absorbante dans le milieu réactif comme du graphite (cf. Fig. 7). En effet, le facteur d'absorption solaire de $\mathrm{CaCO}_{3}$ est faible : $0,16 \pm 0,02$. Dans les conditions optimales, l'efficacité thermochimique $\left({ }^{2}\right)$ est de $20 \%$ à $80 \%$ de décomposition.

Dans le four rotatif, le taux de décarbonatation $(X)$ maximal est de $60 \%$ en régime continu. Si $X$ diminue

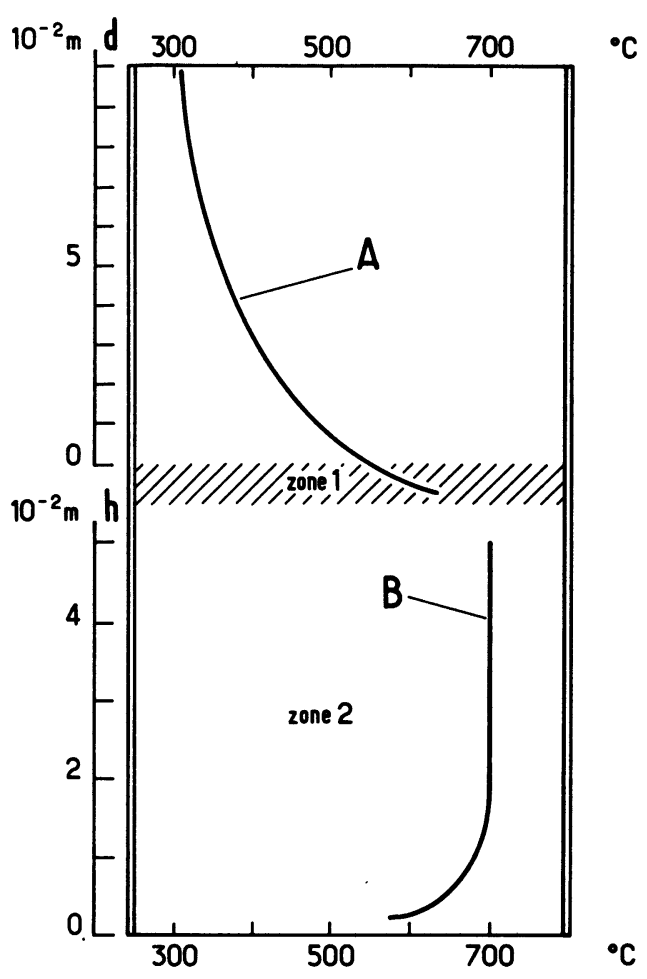

Fig. 4. - Profil de température du gaz au-dessus de la couche (A) et dans la couche fluidisée, dans la zone 2 (B). $u_{0}=0,20 \mathrm{~m} / \mathrm{s}$, sable naturel. Zone $1:$ zone de pénétration du rayonnement concentré $\simeq 6 \times 10^{-3} \mathrm{~m}$. Zone $2:$ zone où les mesures par thermocouple sont possibles.

[Temperature profiles in the fluidized silica sand (thermocouple measurements). Curve A : gas temperature. Curve B : bed temperature.]

régulièrement quand le débit d'alimentation de poudre et la vitesse de rotation du four croissent, la vitesse de décomposition passe par un maximum en fonction du taux de remplissage $\tau^{*}$ calculé par :

$$
\tau^{*}=\frac{\dot{m} t_{\mathrm{s}}^{\prime}}{V \rho_{\mathrm{a}}}
$$

$\dot{m}=$ débit massique d'alimentation,

$t_{\mathrm{s}}=$ temps de séjour moyen de la charge,

$V=$ volume du four,

$\rho_{\mathrm{a}}=$ densité apparente de $\mathrm{CaCO}_{3}$.

Ainsi le taux de remplissage optimal déterminé expérimentalement est $15 \%$. Dans ces conditions, la vitesse de décomposition est de $1,5 \mathrm{~g} / \mathrm{min}$. et l'avancement de la réaction de $30 \%$.

$\left({ }^{2}\right)$ L'efficacité thermochimique (décarbonation) est définie par

$$
\eta_{\mathrm{t}}=\frac{m\left(C_{\mathrm{p}} \Delta T+X \Delta H\right)}{\Delta t P_{\mathrm{u}}}
$$

rapport de la chaleur massique de la charge à la température de réaction plus l'enthalpie de réaction pour un taux de conversion $X$ du matériau à l'énergie utile fournie pendant le temps $\Delta t$, avec $\Delta H=40 \mathrm{kcal} / \mathrm{mole}$ à $900^{\circ} \mathrm{C}$. 


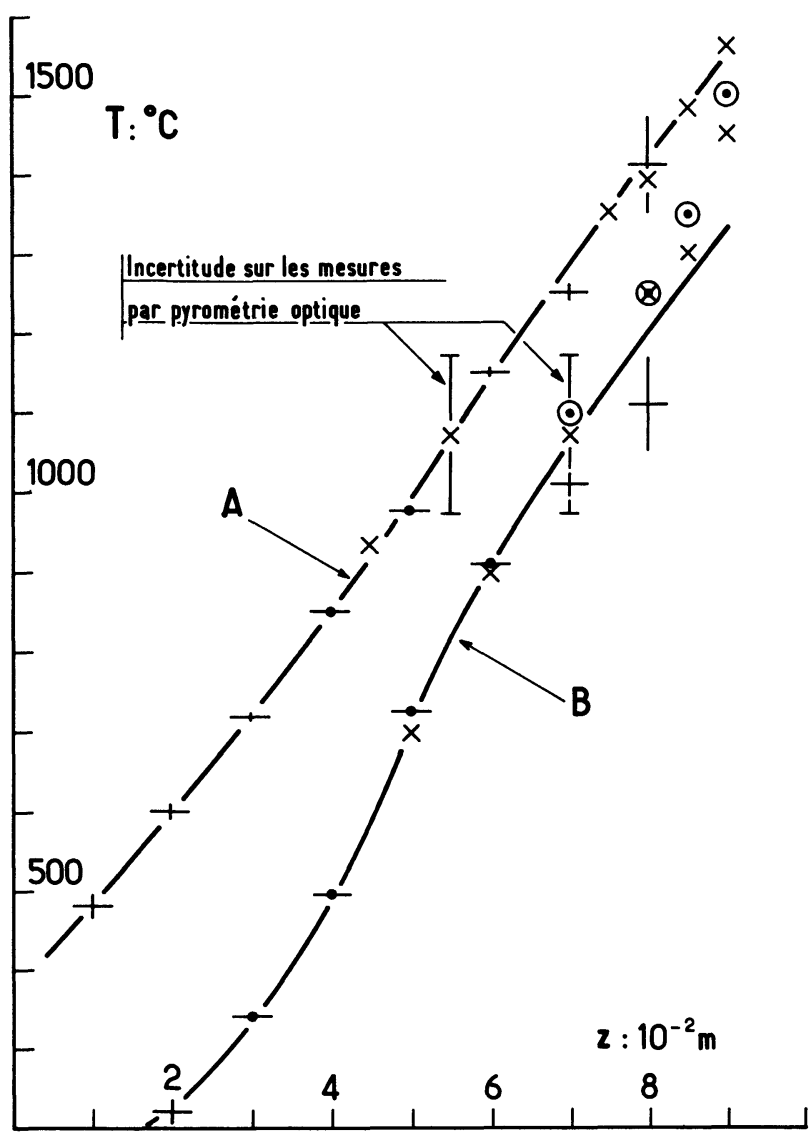

Fig. 5. - Four tournant. Profil axial de température à la surface des tubes en alumine (A) $\alpha_{\mathrm{s}}=0,15, \varnothing=2 \mathrm{~cm}$ et en chromite de lanthane (B) $\alpha_{\mathrm{s}}=0,95, \varnothing=2,4 \mathrm{~cm}$. Comparaison des mesures par thermocouples $(+)$ et des mesures par pyrométrie optique à $\lambda_{1}=0,63 \times 10^{-6} \mathrm{~m}(\odot)$ et $\lambda_{2}=2,9 \times 10^{-6} \mathrm{~m}(\times)$. Four vide : $P_{\mathrm{u}}=1,8 \mathrm{~kW}$.

[Temperature distribution of the rotary kiln walls. Curve A : alumina wall $\alpha_{\mathrm{s}}=0.15 ; \varnothing=2 \mathrm{~cm}$. Curve B : lanthanum chromite wall, $\alpha_{\mathrm{s}}=0.95 ; \varnothing=2.4 \mathrm{~cm} .+$ : thermocouple measurements, $\odot, \times$ : measurements with optical monochromatic pyrometers at $\lambda_{1}=0.63 \mu \mathrm{m}(\odot)$ and $\lambda_{2}=2.9 \mu \mathrm{m}(\times), P_{\mathrm{u}}=1.8 \mathrm{~kW}$.]

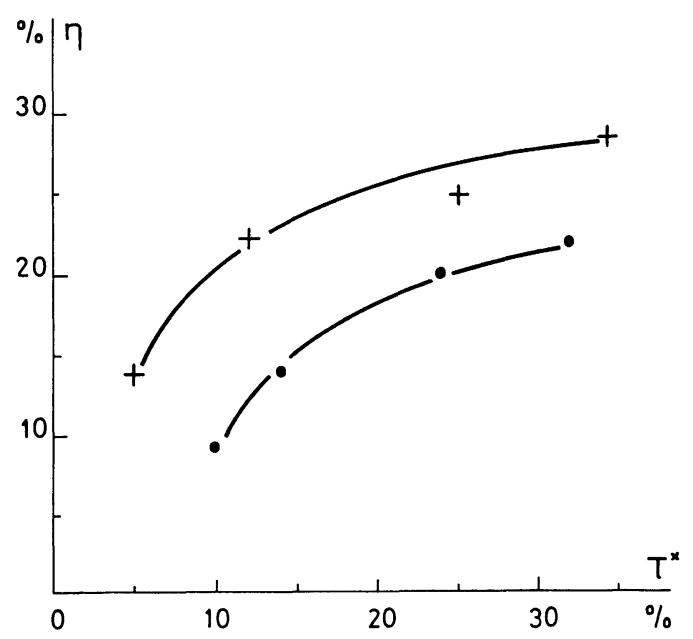

Fig. 6. - Evolution expérimentale de l'efficacité thermique du four rotatif en fonction du taux de remplissage $\tau^{*}$ pour un fonctionnement en régime continu avec du sable naturel. $\bullet \mathrm{Pu}=800 \mathrm{~W}$. $+\mathrm{Pu}=1200 \mathrm{~W}$.

[Experimental variation of the thermal efficiency versus filling ratio for continuous operation with silica sand in the rotary kiln.]
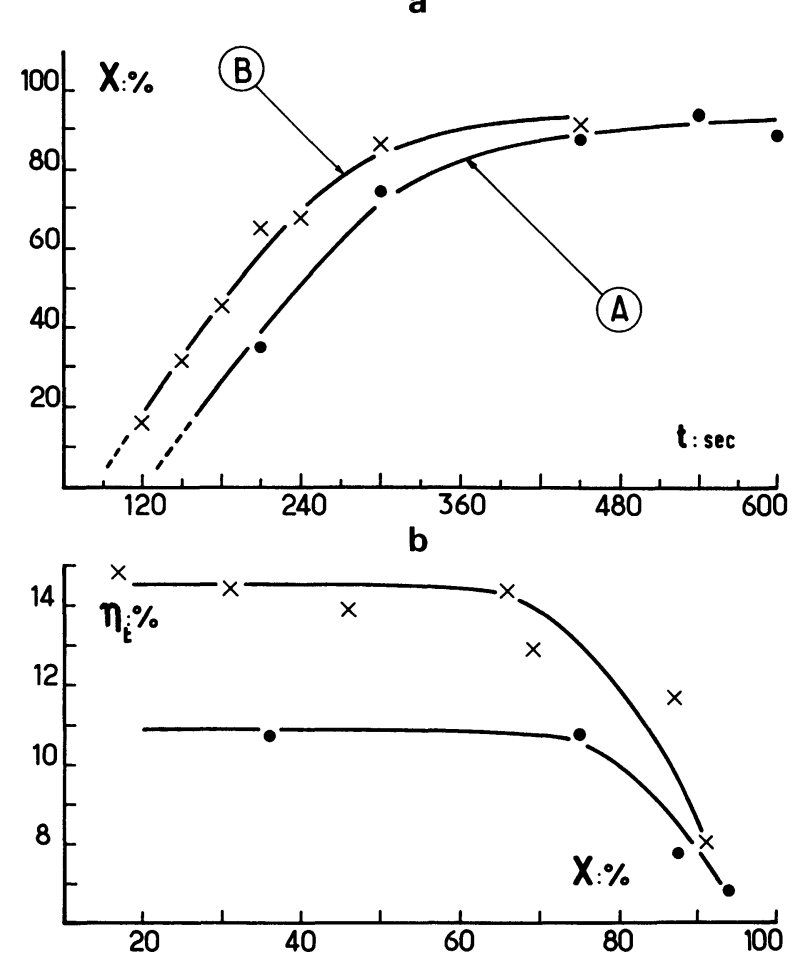

Fig. 7. - Influence du dopage de la charge sur l'avancement $(X)$ et l'efficacité thermo-chimique de la décarbonatation en couche fluidisée. Courbe A : $\mathrm{CaCO}_{3}$ seul. Courbe B : $\mathrm{CaCO}_{3}+1 \%$ de graphite (en poids). $\mathrm{CaCO}_{3}$ masse $=25 \times 10^{-3} \mathrm{~kg}$; distributeur $\varnothing=2 \times 10^{-3} \mathrm{~m} ; 200<d p<315 \times 10^{-6} \mathrm{~m}$; puissance incidente : $P_{\mathrm{u}}=1,4 \mathrm{~kW}$; vitesse superficielle du gaz $: u_{0}=3,3 \times 10^{-2} \mathrm{~m} / \mathrm{s}$.

[Influence of modification of absorptance on reaction ratio and thermal efficiency for the decarbonatation of $\mathrm{CaCO}_{3}$. Curves : $a$ ) Conversion ratio versus time of reaction. $b$ ) Thermo chemical efficiency of decarbonatation versus conversion ratio. Curve A : without addition. Curve B : with addition of $1 \%$ of graphite (by weight) respectively. $P_{\mathrm{n}}=1.4 \mathrm{~kW}$ : beads diameter : $\varnothing=2 \mathrm{~mm}$; particles diameter : $d p=0.200-0.315 \mathrm{~mm}$; weight of the load $m=25 \mathrm{~g} ;$ superficial gas velocity $u_{0}=3.3 \times 10^{-2} \mathrm{~m} / \mathrm{s}$.]

\subsection{CONCLUSION DE L'ÉTUDE EXPÉRIMENTALE. -} Nous avons réuni dans le tableau II les résultats essentiels obtenus avec les deux réacteurs solaires.

Tableau II. - Tableau récapitulatif des résultats.

\section{Caractéristiques}

Facteur d'absorption du système Niveau de température de la charge

Gradient thermique

Efficacite de conversion thermique

Taux de décarbonatation maximal atteint pour $\mathrm{CaCO}_{3}$

Efficacité de conversion pour la décarbonatation de $\mathrm{CaCO}_{3}$

Consommation énergétique (par $\mathrm{kg}$ de $\mathrm{CaO}$ )

$$
\begin{array}{cc}
\begin{array}{c}
\text { Réacteur à lit } \\
\text { fluidisé }
\end{array} & \begin{array}{c}
\text { Four } \\
\text { tournant }
\end{array} \\
\overline{0,5-1} & -
\end{array}
$$

600-1 $300{ }^{\circ} \mathrm{C}$ $200{ }^{\circ} \mathrm{C} / \mathrm{cm}$ sur 1 à $1,5 \mathrm{~cm}$ de hauteur, nul après

$$
\begin{array}{cc}
20-40 \% & 10-30 \% \\
100 \% & 60 \% \\
20 \% & 7 \%
\end{array}
$$

régime discon- régime continu tinu

$9 \mathrm{kWh} / \mathrm{kg}$

$63 \mathrm{kWh} / \mathrm{kg}$ 
Les propriétés d'absorption et d'émission du réacteur à lit fluidisé dépendent des matériaux qui constituent les couches, tandis que le facteur d'absorption du four rotatif est voisin de l'unité quelle que soit la nature de la paroi : alumine $\left(\alpha_{\mathrm{s}}=0,15\right)$ ou chromite de lanthane $\left(\alpha_{\mathrm{s}}=0,95\right)$. Les caractéristiques des deux réacteurs sont très différentes tant au plan thermique que chimique. Si le lit fluidisé est quasi isotherme dans $80 \%$ du volume, un gradient thermique important existe sur toute la longueur des garnissages réfractaires du four rotatif. La moitié arrière du four étant à moins de $900^{\circ} \mathrm{C}$, on peut penser que cette zone est morte au plan chimique, ce qui expliquerait la faible efficacité du four pour la décarbonatation. Comme la distribution du temps de séjour des particules dans les deux réacteurs est très différente - proche du réacteur parfaitement agité en couches fluidisées et du réacteur piston pour le four tournant. Ces réacteurs solaires sont complémentaires et devraient être développés simultanément.

1.5 AmÉlioration Des RÉACTEURS. - L'établissement des bilans thermiques des deux réacteurs laisse apparaître les principales causes de pertes : par rayonnement pour la colonne de fluidisation, par conduction-convection pour le four rotatif. En conséquence, nous sommes amenés à étudier des dispositifs de fluidisation sans paroi transparente et un four rotatif sans réfrigération à eau. Ces nouveaux réacteurs utilisent des parois de transfert métalliques en acier réfractaire $(\mathrm{C}=0,10 \%, \mathrm{Cr}=25 \%, \mathrm{Ni}=20 \%$, $\mathrm{Si}=2 \%$ ) adapté à un fonctionnement en atmosphère oxydante jusqu'à $1150^{\circ} \mathrm{C}$ [1]. Par exemple, on peut associer les avantages des cavités (piège pour le rayonnement) et celles des lits fluidisés (isothermicité, coefficient de transfert de chaleur élevé) dans un lit fluidisé annulaire schématisé figure 8 et proposé en [9].

2. Perspectives d'utilisations "industrielles" des réacteurs proposés. - 2.1 STOCKAGE THERMIQUE ASSOCiÉ A DES CENTRALES ÉleCTROSOLAIRES. - Parmi les différents types de stockage thermique proposés $[8,14,20]$, les réacteurs solaires gaz-solide étudiés s'adaptent bien au stockage thermique ou chimique avec restitution en lit fluidisé. A titre indicatif, nous avons réuni dans le tableau III quelques valeurs comparatives des capacités de stockage de divers matériaux.

Dans le cas envisagé, la fluidisation peut être effectuée directement dans le récepteur solaire ou (et) au niveau d'un échangeur entre les boucles primaires et secondaires du système thermique.

Les calculs approchés qui suivent permettent de situer les ordres de grandeur des installations nécessaires pour réaliser un stockage de $6 \mathrm{~h}$ associé à une centrale solaire de $10 \mathrm{MWe}$. Nous adopterons la valeur $30 \mathrm{MW}$ pour la puissance thermique de l'installation, soit environ $50000 \mathrm{~m}^{2}$ de surface d'héliostats dans les conditions d'ensoleillement du Sud de la France (cf. § 2.2, introduction).

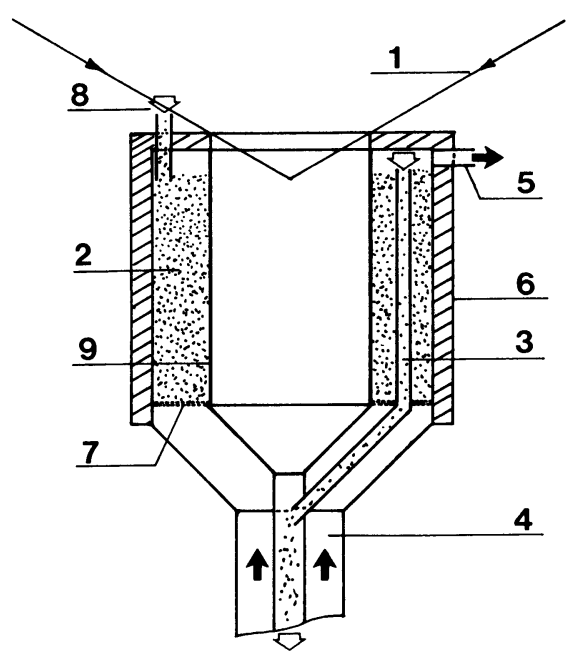

Fig. 8. - Schéma d'un réacteur solaire à lit fluidisé annulaire. 1 : Rayonnement concentré. 2 : Couche fluidisée. 3 : Sortie des particules. 4 : Entrée du gaz vecteur. 5 : Sortie du gaz. 6 : Isolant. 7 : Distributeur du gaz. 8 : Introduction des particules. 9 : Paroi métallique d'après [9].

[Scheme of an annular solar fluid bed reactor. 1 : Concentrated solar rays. 2 : Fluidized bed. 3 : Particules outlet. 4 : Gas inlet. 5 : Gas outlet. 6 : Insulator. 7 : Gas distributor. 8 : Particules inlet. 9 : Metallic transfer wall.]

Tableau III. - Comparaison des capacités de stockage de quelques matériaux d'après $[8,14,20]$.

\begin{tabular}{|c|c|c|c|}
\hline \multirow{3}{*}{ Matériau } & \multirow{3}{*}{$\begin{array}{c}\text { Domaine } \\
\text { de } \\
\text { température } \\
-\end{array}$} & \multicolumn{2}{|c|}{$\begin{array}{c}\text { Capacité de stockage } \\
\text { (thermique) }\end{array}$} \\
\hline & & - & - \\
\hline & & Volumique & Massique \\
\hline 0 - & $100 \quad 250$ & & 11 \\
\hline Terphényl hydrogéné & $100=230$ & & 1,1 \\
\hline (gilotherm) & $100-330$ & 0,5 & 0,6 \\
\hline Sel fondu (HITEC) & $200-450$ & 0,7 & 0,4 \\
\hline Sodium & $150-750$ & 0,23 & 0,24 \\
\hline Sable (lit fluidisé) & $150-1000$ & 0,3 & 0,28 \\
\hline Chaleur latente & ${ }^{\circ} \mathrm{C}$ & $\mathrm{kWh} / \mathrm{m}^{3}$ & $\mathrm{kWh} / \mathrm{t}$ \\
\hline Soude & 320 & 390 & 185 \\
\hline Aluminium & 660 & 310 & 115 \\
\hline Réactions chimiques & & & \\
\hline réversibles & ${ }^{\circ} \mathrm{C}$ & $\mathrm{kWh} / \mathrm{m}^{3}$ & $\mathrm{kWh} / \mathrm{t}$ \\
\hline $\begin{array}{l}\mathrm{SO}_{2}+1 / 2 \mathrm{O}_{2} \rightleftarrows \mathrm{SO}_{3} \\
\left(\mathrm{SO}_{2}, \mathrm{SO}_{3} \text { liq. } ; \mathrm{O}_{2} 100 \mathrm{~b}\right)\end{array}$ & $500-550$ & 70 & 230 \\
\hline $\begin{array}{l}\mathrm{NH}_{3}+\mathrm{H}_{2} \mathrm{O}+\mathrm{SO}_{3} \rightleftarrows \\
\rightleftarrows \mathrm{NH}_{4} \mathrm{HSO}_{4}\end{array}$ & $550-600$ & 140 & 500 \\
\hline $\mathrm{CO}+3 \mathrm{H}_{2} \rightleftarrows$ & & & \\
\hline$\underset{(\operatorname{gaz} 100 \mathrm{~b})}{\rightleftarrows \mathrm{CH}_{4}+\mathrm{H}_{2} \mathrm{O}}$ & 600 & 1 & 6,6 \\
\hline $\mathrm{CaO}+\mathrm{CO}_{2} \rightleftarrows \mathrm{CaCO}_{3}$ & $650-700$ & $\begin{array}{c}35-350 \\
\text { (voir texte) }\end{array}$ & $27-270$ \\
\hline
\end{tabular}

2.1.1 Stockage thermique sur sable associé à un cycle ouvert à gaz. - A moyenne température (500$700^{\circ} \mathrm{C}$ ) des dispositifs de stockage en lits fluidisés ont été proposés en association à des cycles à vapeur [4] ou à hélium [3]. Par ailleurs, des études sont actuel- 
lement en cours au four solaire de $1000 \mathrm{~kW}$ en association avec l'Institut du Génie Chimique de Toulouse en vue du chauffage et du stockage des solides granulaires à haute température [15]. Enfin, nos propres résultats ont permis de montrer qu'il est possible de chauffer un solide divisé vers $1000^{\circ} \mathrm{C}$ par rayonnement solaire concentré. Il semble donc intéressant de chauffer le gaz et le solide dans un réacteur solaire à lit fluidisé, de détendre d'une part le gaz chaud dans une turbine et de stocker d'autre part le solide à haute température $\left(800-900^{\circ} \mathrm{C}\right)$ dans un silo. Le dispositif proposé est schématisé figure 9 . Il se décompose comme suit :

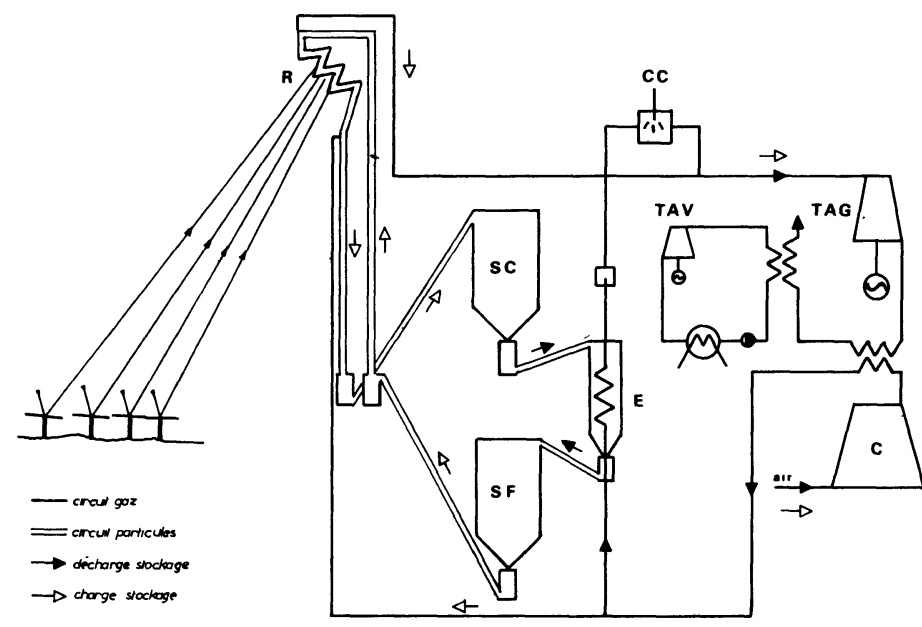

Fig. 9. - Représentation schématique d'une centrale électrosolaire à récepteur à lit fluidisé utilisant un cycle ouvert à gaz et un stockage thermique sur particules réfractaires.

[Scheme of a solar power plant using a fluidized bed receiver, an opened gas cycle and a refractory particles thermal storage.]

- Le réacteur à lit fluidisé : $\mathrm{R}$, placé au sommet de la tour de la centrale solaire est alimenté en solide par le stock froid - SF - Les grains chauds sont convoyés vers le stock chaud - SC - tandis que le gaz de fluidisation est envoyé dans la turbine à gaz - TAG - . Le compresseur - C - alimente en air soit le réacteur, soit l'échangeur à lits fluidisés - E pendant les périodes non ensoleillées. Dans ce cas, l'échangeur est alimenté en particules chaudes venant de - SC - et libère le solide froid dirigé vers - SF - La chambre de combustion - CC - permet le démarrage de l'installation et sert de chaudière d'appoint quand la température du stockage est trop basse. L'addition éventuelle d'un cycle à vapeur - TAV - en queue permet de réduire les pertes thermiques dans le gaz d'échappement de la turbine à gaz. Le rendement thermodynamique de ce type d'installation pourrait alors atteindre 35 à $40 \%[13,16,19]$.

Pour un stock chaud à $900^{\circ} \mathrm{C}$, un stock froid à $400^{\circ} \mathrm{C}$ et une turbine fonctionnant entre 900 et $400^{\circ} \mathrm{C}$, les principales caractéristiques de l'installation sont :
- Réacteur. - Surface exposée au flux solaire : 200 à $400 \mathrm{~m}^{2}$. D'après Granier [12] les coefficients de transfert lit fluidisé-paroi sont d'environ $400 \mathrm{~W} / \mathrm{m}^{2}{ }^{\circ} \mathrm{C}$ à basse température. On peut espérer atteindre des valeurs de 800 à $900 \mathrm{~W} / \mathrm{m}^{2}{ }^{\circ} \mathrm{C}$ vers $900{ }^{\circ} \mathrm{C}$.

- Stockage du sable. - La masse nécessaire pour stocker $200 \mathrm{MWth}$ est de $1700 \mathrm{t}$, soit $1550 \mathrm{~m}^{3}$. Ce volume est contenu dans un cylindre de $10 \mathrm{~m}$ de diamètre et $20 \mathrm{~m}$ de haut.

- Echangeur

- Débit de solide : $275 \mathrm{t} / \mathrm{h}$.

- Débit d'air - $214 \mathrm{t} / \mathrm{h}\left(100000 \mathrm{~m}^{3} / \mathrm{h}\right.$ pour une pression d'admission de la turbine de 7 bar).

Pour une vitesse de fluidisation de $1 \mathrm{~m} / \mathrm{s}$ le diamètre du lit serait de $6 \mathrm{~m}$.

2.1.2 Stockage chimique par le système $\mathrm{CaCO}_{3} /$ $\mathrm{CaO} . \mathrm{CO}_{2}$ associé à un cycle fermé à hélium. - Par rapport au stockage par chaleur sensible, le stockage chimique permet la restitution des calories sur un palier de température et généralement un volume du stock réduit.

Le schéma de l'installation proposée est représenté figure 10 : le réacteur $\mathrm{R}$ (lit fluidisé ou four rotatif) est alimenté en calcite $\mathrm{CaCO}_{3}$ venant du stock froid. La chaux produite est accumulée dans le stock chaud, tandis que le gaz chaud traverse l'échangeur - EGGavant d'être stocké dans la capacité $-\mathrm{S} \cdot \mathrm{CO}_{2}-\mathrm{La}$ source chaude du cycle fermé à hélium est, soit l'échangeur - EGG - , soit l'échangeur - EGS A l'intérieur de ce dernier est réalisée la réaction exothermique $\mathrm{CaO}+\mathrm{CO}_{2} \rightarrow \mathrm{CaCO}_{3}$ en couches fluidisées multiétagées vers $700^{\circ} \mathrm{C}$. Au sein des lits fluides, l'hélium circule dans des serpentins. Ce dispositif. permet d'atteindre des coefficients de transfert globaux de l'ordre de $350 \mathrm{~W} / \mathrm{m}^{2}$. ${ }^{\circ} \mathrm{C}$ [3]. L'échangeur - EGS - est alimenté en solide par - S.CaO et en mélange gazeux air $/ \mathrm{CO}_{2}$ par le compresseur $-\mathrm{Cl}$ -

Le prédimensionnement d'une telle installation est délicat étant donné l'incertitude existant sur la capacité réelle de stockage volumique du système $\mathrm{CaO} /$ $\mathrm{CaCO}_{3}$. En effet Barker [2], sur la base de données expérimentales indique qu'elle varie de $35 \mathrm{kWh} / \mathrm{m}^{3}$ à $350 \mathrm{kWh} / \mathrm{m}^{3}$. Pour une valeur moyenne de $200 \mathrm{kWh} / \mathrm{m}^{3}$, le volume du stock serait d'environ $1000 \mathrm{~m}^{3}$, soit $800 \mathrm{t}$.

Cette estimation montre que le volume des silos est nettement plus faible que pour le stockage sur sable. Néanmoins, il faut y ajouter la capacité de $\mathrm{CO}_{2}$ sous pression. Afin d'éviter ce dispositif, on peut envisager le couplage de l'installation avec une centrale électrique à charbon, ou fuel, permettant d'utiliser les gaz de combustion produits dans ces unités (contenant une forte proportion de $\mathrm{CO}_{2}$ ), comme source de dioxyde carbone pour le réacteur échangeur de carbonatation de la chaux. 


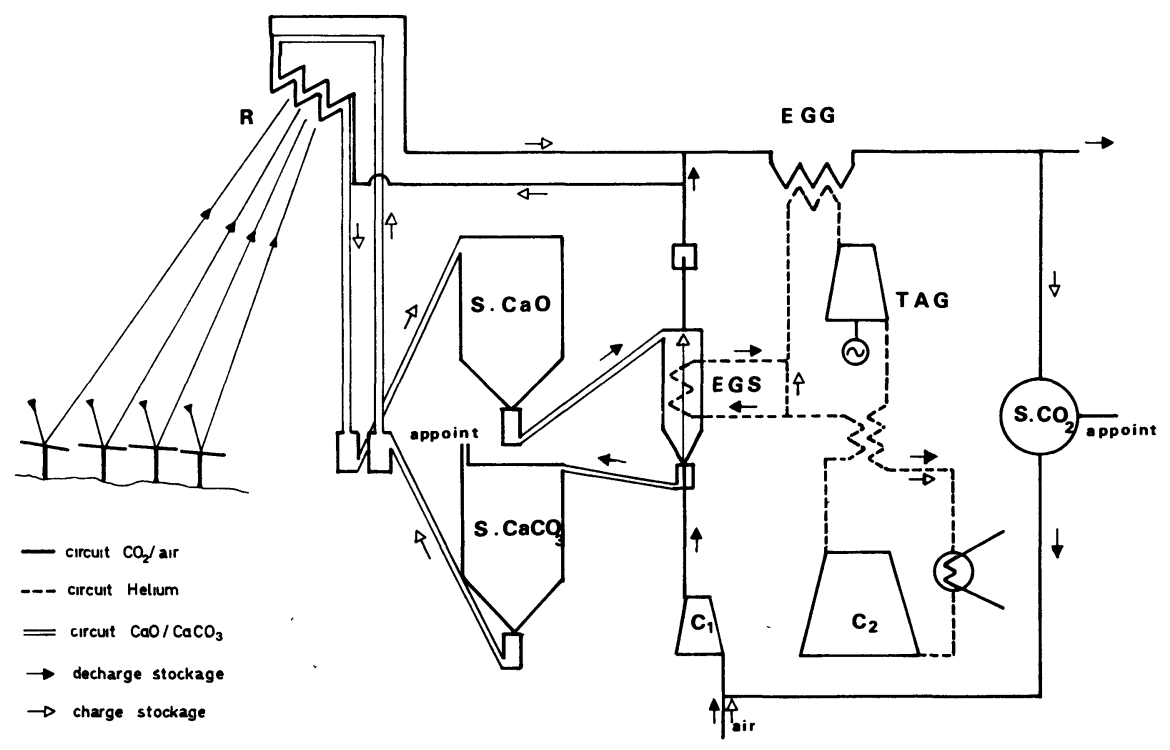

Fig. 10. - Représentation schématique d'une centrale solaire associée à un cycle fermé à hélium et à un stockage chimique par le système $\mathrm{CaO}+\mathrm{CO}_{2} / \mathrm{CaCO}_{3}$.

[Scheme of a closed gas cycle solar power plant with a chemical storage : the system $\mathrm{CaO}+\mathrm{CO}_{2} / \mathrm{CaCO}_{3}$.]

En ce qui concerne le cycle à hélium, Griepentrog $\mathrm{A}$. [13] donne les caractéristiques d'une turbine à hélium de 13,75 MWe fonctionnant à Oberhausen à un niveau de température compatible avec celui escompté pour la réaction de carbonatation de la chaux :

température d'admission au compresseur : $20{ }^{\circ} \mathrm{C}$, pression d'admission : 8,15 bar,

température d'admission à la turbine : $720^{\circ} \mathrm{C}$,

pression d'admission : 32,6 bar,

rendement thermodynamique : $29,5 \%$ (de 50 à $100 \%$ de la charge).

L'inconvénient majeur du système réside dans l'irréversibilité partielle de la réaction

$$
\mathrm{CaCO}_{3} \rightarrow \mathrm{CaO}+\mathrm{CO}_{2} \text {. }
$$

Après des cycles répétés, Barker observe une réactivité de $93 \%$ après 30 cycles mais pour un matériau très fin. Pour y remédier, il semble attrayant d'utiliser le double intérêt de la chaux (moyen de stockage et matériau de construction) : lorsque la réactivité de $\mathrm{CaO}$ baisse, la revendre en vue de la fabrication $\mathrm{du}$ ciment par exemple.

2.1.3 Technologie des procédés. - On peut dégager de ces propositions quelques problèmes technologiques cruciaux qui restent à résoudre, au moins partiellement, et dont dépend le bon fonctionnement des cycles :

- Tenue mécanique des matériaux soumis à des chocs thermiques répétés dus à l'alternance journuit et aux passages nuageux. En particulier le récepteur solaire, dont les parois exposées au flux solaire seraient à environ $1000^{\circ} \mathrm{C}$, inclinées et sous pression, est un des composants sensibles des systèmes.
- Convoyage du solide chaud entre le récepteur, le stockage chaud et l'échangeur. Le transport pneumatique semble le plus adapté, mais il est assez gros consommateur d'énergie mécanique.

- Pertes énergétiques de l'ensemble du cycle : thermique (parois à $900^{\circ} \mathrm{C}$ ) et mécaniques (compression, transport de solide).

- Dans le cas particulier de la réaction gaz-solide, on peut ajouter qu'il conviendrait de développer une étude précise de la réversibilité et de la cinétique de la réaction de carbonatation.

Cette énumération, non exhaustive, montre qu'il reste un gros effort de recherche et développement à fournir concernant les technologies à hautes températures pour proposer des projets pilotes viables.

\subsection{FABRICATION DE LIANTS HYDRAULIQUES A PAR-} TIR D'INSTALLATIONS SOLAIRES A RÉCEPTEUR CENTRAL. - Le prix du $\mathrm{kWh}$ fourni par une centrale solaire installée en zone tempérée méditerranéenne est estimé à une valeur dix fois supérieure à celui délivré par une centrale électrique classique. Par contre, l'écart serait beaucoup plus faible lorsqu'on s'intéresse à la valorisation directe de la thermie solaire. En France, l'écart entre le prix de la thermie solaire et celui de la thermie gaz ou fuel ne serait plus que d'un facteur 5 (taux d'actualisation de $10 \%$ sur 30 ans) et serait réduit à 3 en zone subtropicale [5]. Dans ces conditions, l'utilisation du rayonnement solaire concentré en vue de l'élaboration de liants hydrauliques à moyenne et haute température semble intéressante, ces procédés de fabrication mettant en œuvre des réactions très endothermiques : par exemple, $40 \mathrm{kcal} / \mathrm{mole}$ pour décarbonater $\mathrm{CaCO}_{3}$ à $900{ }^{\circ} \mathrm{C}$. 
Dans les exemples qui suivent, nous considérons un module de $30 \mathrm{MWt}$ implantée dans le sud de la France. Nous avons estimé que cette installation fonctionne en moyenne $1300 \mathrm{~h}$ par an. Cette durée correspond à l'ensoleillement annuel moyen supérieur ou égal au seuil de $500-600 \mathrm{~W} / \mathrm{m}^{2} \mathrm{~d}$ 'après les données relevées à Odeillo [18].

2.2.1 Production de chaux et de ciments pouzzolaniques. - Le bilan thermique de la production d'une tonne de chaux s'établit comme suit :

chaleur sensible à $900^{\circ} \mathrm{C}: 1370 \mathrm{MJ}$,

chaleur de réaction à $900^{\circ} \mathrm{C}: 2790 \mathrm{MJ}$.

$\mathrm{Si}$ on admet une récupération à $80 \%$ de la chaleur sensible entre $\mathrm{CaO}-\mathrm{CO}_{2}$ sortant du réacteur et $\mathrm{CaCO}_{3}$ entrant, les besoins théoriques sont :

pertes : $275 \mathrm{MJ}$,

réaction : $2790 \mathrm{MJ}$,

soit $3065 \mathrm{MJ} / \mathrm{t}$ ou $850 \mathrm{kWht} / \mathrm{t}$ de chaux. La capacité de production de l'installation solaire envisagée (pour un rendement thermique du réacteur de $60 \%$ ) serait d'environ $27500 \mathrm{t} /$ an de chaux.

- En ce qui concerne les ciments pouzzolaniques, le bilan thermique approximatif est :

activation de l'argile à $600-700{ }^{\circ} \mathrm{C}: 350 \mathrm{kWht} / \mathrm{t}$, fabrication de la chaux : $850 \mathrm{kWht} / \mathrm{t}$,

soit une production annuelle de $20000 \mathrm{t}$ de ciments pouzzolaniques.

Ces procédés impliquent un important dégagement de dioxyde de carbone. Nous avons envisagé d'en récupérer la chaleur sensible pour préchauffer le solide entrant, certains auteurs ont proposé la valorisation chimique de cette molécule. On peut citer : la production d'hydrocarbures synthétiques via le méthanol [17] et la synthèse de l'hydrogène par un procédé hybride (thermique et électrolytique) en utilisant l'acide formique comme intermédiaire de réactions [22].

2.2.2 Production de ciment "Portland». - La consommation énergétique théorique par tonne de clinker est de $515 \mathrm{kWht}$ pour le procédé par voie sèche (il faut 1,56 $\mathrm{t}$ de cru par tonne de ciment) et d'environ $920 \mathrm{kWht}$ dans la pratique. L'industrie du ciment est donc grosse consommatrice d'énergie puisque pour une consommation annuelle de 35 millions de tonnes, la France dépense environ 3 MTEP pour assurer cette production (soit près de $1,5 \%$ de l'énergie totale consommée annuellement en France).

Les étapes successives de la fabrication sont [11] :

1) Préchauffage $20-700{ }^{\circ} \mathrm{C}$.

2) Décarbonatation $700-900{ }^{\circ} \mathrm{C}$.

3) Chauffage jusqu'à $1350^{\circ} \mathrm{C}$.

4) Clinkérisation $1350-1450^{\circ} \mathrm{C}$.

5) Refroidissement avec récupération des calories.

L'installation projetée ne permettrait pas la réalisation d'une réaction à $1400^{\circ} \mathrm{C}$. Il faut alors envi- sager soit de la doubler d'un dispositif à double réflexion (type four solaire parabolique), soit d'effectuer les étapes 3 et 4 selon les procédés classiques. Nous adopterons cette seconde solution.

Le procédé consisterait donc à fournir la chaleur nécessaire aux réactions endothermiques 1 et 2 par voie solaire, tandis que l'étape 4 serait effectuée de façon classique en four rotatif. La clinkérisation étant exothermique, un récupérateur de chaleur entre 3 et 4 permettrait de fournir la quasi-totalité de la chaleur sensible nécessaire au chauffage de la charge entre 900 et $1350^{\circ} \mathrm{C}$. Le procédé serait ainsi presque entièrement solaire. Pour éviter la succession de régime transitoire dans le four classique à haute température, les deux unités décrites précédemment seraient couplées par un stock de $c r u$ décarbonaté à $900^{\circ} \mathrm{C}$ (permettant le fonctionnement en continu de la partie classique). A titre indicatif, un silo de $500 \mathrm{~m}^{3}$ (environ $650 \mathrm{t}$ de $\mathrm{CaO}$ ) à $800^{\circ} \mathrm{C}$ composé d'une enveloppe externe en béton et d'un revêtement interne en briques réfractaires isolantes (de $20 \mathrm{~cm}$ d'épaisseur chacun) ne perd que $8{ }^{\circ} \mathrm{C} / \mathrm{j}$ sur l'axe et $25^{\circ} \mathrm{C} / \mathrm{j}$ de la paroi [6].

\section{Bilan thermique approximatif :}

- Partie solaire :

déshydratation : $135 \mathrm{MJ} / \mathrm{t}$, décarbonatation : $2180 \mathrm{MJ} / \mathrm{t}$, chaleur sensible du cru : $800 \mathrm{MJ} / \mathrm{t}$, chaleur sensible du $\mathrm{CO}_{2}$ dégagé : $630 \mathrm{MJ} / \mathrm{t}$, si on suppose une récupération à $80 \%$, les pertes s'élèvent à $130 \mathrm{MJ} / \mathrm{t}$ (pertes dans les fumées). On a donc théoriquement $3250 \mathrm{MJ} / \mathrm{t}$ (environ $900 \mathrm{kWht} / \mathrm{t}$ ) à fournir par voie solaire.

- Partie classique : il faut que la chaleur dégagée au cours de la clinkérisation compense les besoins thermiques du four : pertes et chaleur sensible du cru entre 850 et $1350^{\circ} \mathrm{C}$.

Exothermicité de la clinkérisation : $460 \mathrm{MJ} / \mathrm{t}$;

Chaleur sensible $850-1350^{\circ} \mathrm{C}: 470 \mathrm{MJ} / \mathrm{t}$;

Pertes (fumées + parois) : $730 \mathrm{MJ} / \mathrm{t}$,

d'où une consommation en combustible fossile de $720 \mathrm{MJ} / \mathrm{t}(200 \mathrm{kWht} / \mathrm{t})$. Le clinker sortant à $1400^{\circ} \mathrm{C}$, un récupérateur de chaleur couplé à une turbine peut ensuite fournir en électricité les auxiliaires de la cimenterie.

\section{Production annuelle}

Pour un rendement thermique de la partie solaire de $60 \%$, la productivité est d'environ $25000 \mathrm{t}$ /an de ciment. Les caractéristiques principales de l'installation sont dans ces conditions :

Capacité du four de clinkérisation : 3-4 t/h ;

Capacité du stockage : 800-1500 t;

Consommation de combustible fossile : $400 \mathrm{t} / \mathrm{an}$;

Economie de combustible fossile : environ $2000 \mathrm{t} / \mathrm{an}$.

Comparée à la capacité de production d'une cimenterie classique $-1000 \mathrm{t} / \mathrm{j}$ en moyenne, soit environ 
$350000 \mathrm{t} / \mathrm{an}$ - cette unité solaire a une production assez faible. L'émergence de tels procédés ne peut donc pas s'inscrire dans le cadre de la course au gigantisme industriel actuel (cimenterie de $5000 \mathrm{t} / \mathrm{j}$ au Japon), mais dans un processus de développement basé sur les ressources renouvelables qui pourrait être encouragé dans les pays en développement.

3. Conclusion. - Après avoir démontré la faisabilité, en régime continu et discontinu, de réactions chimiques endothermiques vers $1000^{\circ} \mathrm{C}$ par voie solaire, nous avons proposé quelques applications à grande échelle des réacteurs étudiés. Les possibilités d'applications sont nombreuses, tant dans le domaine énergétique (turbines à gaz) que chimique (liants hydrauliques, réactions catalytiques...). Un vaste champ d'investigation reste encore à développer en ce qui concerne : l'étude des réacteurs (amélioration des rendements thermiques, l'étude des matériaux soumis à des cycles répétés de température - plusieurs milliers par an - et des transferts d'énergie en présence de rayonnement), la conception d'installations à énergie et chimie totale, la réduction du coût des composants.

Dans un pays industrialisé européen, l'impact des projets proposés semble encore faible : économie de $2000 \mathrm{TEP} /$ an par cimenterie de $30 \mathrm{MW}$ th, il faudrait plus de 130 de ces unités pour produire $10 \%$ de la consommation française annuelle de ciment. Par contre, il conviendrait d'étudier l'insertion de ces -installations dans l'économie de pays en développement, souvent très bien ensoleillés et pourvus en matières premières.

\section{Bibliographie}

[1] Badie, J. M., Bonet, C., Faure, M., Flamant, G., Foro, R., HeRnANDEZ, D., " Decarbonation of calcite and phosphate rock in solar chemical reactors » à paraître dans Chem. Eng. Sci.

[2] Barker, R., J. Appl. Chem. Biotechnol. 24 (1974) 221-227.

[3] Bergougnou, M. A., Large, J. F., Rev. Gen. Therm., Fr. 206 (1979) 87-96.

[4] Botterill, J. S. M., VirR, M. J., Appl. Energy, 3 (1977) 139-150.

[5] Claverie, M., Etievant, C., Revue de l'Energie, 313, Special Energie Solaire (1979) 200-208.

[6] Derouette, J. J., Dartoy, J., Perspective d'utilisation de l'énergie solaire dans les unités de fabrication du méthanol, Rapport de l'Institut Battelle (Genève) par le CNRS, nov. 76.

[7] ERvin, G., J. solid state chem. 22 (1977) 51-61.

[8] Etievant, Peube, Vialaron, Allard, Bonnin, Pharabod. Colloque International Electricité Solaire, Toulouse, 1-5 mars 1976, 595-612. Ed. Imprimerie du Sud. Toulouse.

[9] Flamant, G., Thèse Docteur Ingénieur no 311, Université Paul-Sabatier, Toulouse, 20 octobre 1978.

[10] Flamant, G., Bonet, C., Hernandez, D., Traverse, J. P., Revue Internationale d'héliotechnique (COMPLES) $2^{\mathrm{e}}$ semestre 1977, 39-43.

[11] Ghestern, G., Herriquet, P., Ciments, bétons, plâtres, chaux, no 1 (1978) 20-25.
[12] Granier, M., Couderc, J. P., Echangeurs de chaleur à plaques à couche fluidisée. Journées sur la technologie der lits fluidisés et dispersés, Compiègne, 22-23 octobre 1979.

[13] Griepentrog, H., Entropie, 79 (1978) 18-28.

[14] Journée d'Etude de la S.E.E. Stockage de l'énergie et électricité, 2-3 juin 1977. Réf. 7711 262. Notamment Communication de Messieurs BonIN et PACAUlT.

[15] Royère, C., Rapport au Comité des Utilisateurs du four solaire de $1000 \mathrm{~kW}$ du CNRS, 23 novembre 77 et au S.T.T.F.U.A., Albuquerque, 3-4 mai 1979.

Royère, C., LAGUERIE, C., « Captation, stockage et restitution d'énergie thermique à haute température pour l'utilisation du rayonnement solaire concentré à l'aide d'un caloporteur solide divisé. " A.T.P. Mécanique et Thermodynamique, $\mathrm{n}^{\circ} 3938,26$ juillet 78.

[16] Sawyer's gas turbine engineering handbook. Ed. J. W. Sawyer (1972).

[17] SteinberG, M., Fuel, Vol. 57 (1978) 460-468.

[18] Tricaud, J. F., Atlas Energétique du rayonnement solaire pour la France, Pyc. Edition (1978).

[19] Vandamme, E., Borin, R., Rev. Gen. Therm. Fr nos 198-199, juin-juillet 79 .

[20] Vialaron, A., Revue Internationale d'Héliotechnique, p. 38-41. 2e semestre 1978.

[21] Villermaux, Entropie, 85 (1979) 25-31.

[22] Williams, R., Grandall, R. S., Bloom, A., Appl. Phys. Lett. 33 (5) (1978) 381-383. 